\title{
Pronóstico a corto plazo en pacientes con ataques isquémicos transitorios
}

Short-term prognosis after Emergency Department diagnosis of TIA. Jhonston CS, Gress DR, Browner WS, Sidney S. JAMA 2000; 284: 2901-2906.

\section{Objetivo}

Determinar el riesgo de accidente cerebrovascular (ACV) a corto-plazo y de otros eventos adversos luego del diagnóstico de accidente isquémico transitorio (TIA).

\section{Diseño}

Estudio de cohorte prospectiva*

\section{Lugar}

Dieciseis hospitales pertenecientes a la Kaiser-Permanente de California (Sistema de Salud Gerenciado).

\section{Pacientes}

Desde marzo 1997 hasta febrero 1998, se identificó a los pacientes con diagnóstico de TIA evaluados en la guardia. Para obtener la información médica se utilizó su historia clínica, evaluada por un neurólogo que desconocía la evolución de los pacientes. Se excluyeron los pacientes que no tuviesen disponible la historia de guardia $(n=30)$, miembros fuera de la Kaiser $(n=27)$, aquéllos codificados con otros diagnósticos $(n=25)$ y los pacientes con TIA previo $(n=8)$.

\section{Evaluación de factores de riesgo}

Se evaluaron variables demográficas, clínicas, factores de riesgo vascular y varios hallazgos del examen físico (FC, presencia de soplos vasculares, debilidad, parestesias, confusión y alteraciones del lenguaje).

\section{Evaluación de resultados principales}

Se utilizaron cuatro resultados primarios: a) Hospitalizaciones, b) ACV, c) Nuevo TIA, d) Muerte. Estos fueron evaluados a los 90 días luego de haber tenido el TIA inicial. El diagnóstico final de
TIA y ACV requería la evaluación de un neurólogo y el diagnóstico médico escrito en la historia clínica.

\section{Resultados Principales}

De 1707 pacientes con TIA, 99\% ingresaron a la guardia dentro de las 24 hs. El promedio de duración de los síntomas fue 207 minutos. Dentro de los 90 días posteriores al TIA 180 pacientes $(10.5 \%)$ tuvieron un ACV, y el $50 \%$ ocurrió dentro de las $48 \mathrm{hs}$ del TIA. Sólo el $7 \%$ de los pacientes con ACV quedaron sin discapacidad a los 90 días. La mortalidad en este grupo fue del $21 \%$. Los factores de riesgo independientes para desarrollar ACV fueron: edad, duración del TIA, síntomas al ingreso, diabetes, debilidad, alteraciones en el lenguaje o en la marcha fueron (ver tabla). Haber recibido tratamiento previamente (aspirina, anticoagulación, etc) no influyó en el pronóstico.

Tabla. Factores de riesgo independientes para ACV a 90 días

\begin{tabular}{l|c|c}
\hline Factor & $\mathbf{O R}^{\boldsymbol{*}} \mathbf{( I C ~ 9 5 \% )}$ & $\mathbf{p}$ \\
\hline Edad $>\mathbf{6 0}$ & $1.8(1.1-2.7)$ & 0.01 \\
\hline Diabetes & $2.0(1.4-2.9)$ & $<0.001$ \\
\hline Caracteristicas del Episodio & & - \\
\hline Duración: $>\mathbf{1 0}$ minutos & $2.3(1.3-4.2)$ & 0.005 \\
\hline Debilidad & $1.9(1.4-2.6)$ & $<0.001$ \\
\hline Dificultad para hablar & $1.5(1.1-2.1)$ & 0.01 \\
\hline
\end{tabular}

\section{Conclusiones:}

El riesgo de ACV a corto plazo en los pacientes que ingresan a una guardia con TIA es sustancial. La identificación de algunos factores puede ayudar en la evaluación y tratamiento precoz de esta patología.

\section{Comentario}

El presente estudio intenta identificar variables clínicas para determinar el riesgo de ACV en pacientes que ingresan a una guardia con un TIA. Su mayor valor reside en estudiar una situación clínica frecuente. Los autores utilizan un seguimiento de 90 días y encuentran que la presencia de un TIA supone un riesgo de desarrollar un ACV del 10\%, cincuenta veces mayor que el esperado para un paciente de similar edad. El riesgo combinado de cualquier evento vascular fue del $25 \%$. De los pacientes que desarrollan un ACV, más de dos tercios quedan con discapacidad, siendo lạ mortalidad superior al $20 \%$.

A pesar de la riqueza de información obtenida y de la estrategia empleada en el análisis, surgen algunas consideraciones. En primer lugar, el diagnóstico de TIA es primariamente clínico ${ }^{1}$ y la dificultad del paciente en explicar los síntomas constituye su principal factor limitante.2 También, la precisión del diagnóstico se reduce cuando es efectuado por médicos no neurólogos,3-5 y la presencia de síntomas aislados (como confusión, vértigo, inestabilidad en la marcha, etc) pueden reflejar otras enfermedades más que constituir una manifestación de enfermedad cerebrovascular. Esto puede ser mejorado, aunque no del todo, por la evaluación del neurólogo. Por otro lado, la utilización de una fuente secundaria, en este caso revisión de historias clínicas, es controvertida cuando el proceso a estudiar es difícil de mensurar (Ej: TIA, dolor abdominal, etc), más aun cuando las historias clínicas no estaban estandarizadas y fueron libremente confeccionadas por médicos no-neurólogos. Aunque los autores intentan corregir en el análisis estas dificultades, no se puede descartar el error sistemático. Por último, aunque los resultados sean probablemente aplicables a nuestro medio, la población en estudio corresponde a pacientes pertenecientes a un sistema prepago de EE.UU.

Como conclusión, el presente estudio utiliza un diseño factible y, a pesar de sus limitaciones, aporta información útil. Lo que los médicos en la guardia diagnostican como TIA tiene un importante impacto en los eventos vasculares a 90 días. Algunas sencillas variables clínicas pueden ser útiles para estratificar el riesgo.

*Ver glosario

\section{Dr. Gustavo Saposnik [ Cordinador Unidad de ACV. Htal JM Ramos Mejía - GCBA. Docente de Neurología de la UBA ]}

Supplement to the Guidelines for the Management of Transient Ischemic Attacks. A Statement From the Ad Hoc Committee on Guidelines for the Management of Transient Ischemic Atacks, ACV Council, American Heart Association. Albers GW, Hart Robert G, Lutsep HL. ACV 1999; 30: 2502-2511

2. Transient ischemic attacks and the general practitioner: diagnosis and management. Quik-van Milligen MLT, Kuyvenhoven MM, de Melker RA. Cerebrovasc Dis. 1992;2:102-106. 3. Diagnosis of Transient ischemic attack by non-neurologist. Ferro JM, Falcao I, Rodrigues G. ACV 1996; 27: 2225-2229. 4. Cooperative study of Hospital frequency and character of trnasient ischemic attacks. IV. The reliability of diagnosis. Calanchini PR, Swanson PD, Gotshall RA. JAMA 1977; 238: 20292033 